\title{
Pulmonary infection with Mycobacterium xenopi: review of treatment and response
}

\author{
J BANKS, AM HUNTER, IA CAMPBELL, PA JENKINS, AP SMITH \\ From the Chest Unit, Llandough Hospital, Penarth; and the Mycobacterium Reference Unit, University \\ Hospital of Wales, Cardiff
}

ABSTRACT Forty seven patients ( $82 \%$ male) with pulmonary infection caused by Mycobacterium $x$ xenopi have been reviewed. Pre-existing lung disease was present in $35(75 \%)$. In 21 patients the disease was characterised by a subacute illness developing over a period of two to four months, while in another 20 patients there was a longer history of chronic respiratory problems often associated with slowly progressive changes evident from chest radiographs. Response to treatment was poor and unpredictable, and was not related to the results of in vitro sensitivity tests, pre-existing lung disease, or mode of onset of symptoms. Eleven patients $(23 \%)$ were cured with chemotherapy. The best drug regimen appeared to be rifampicin and isoniazid combined with either streptomycin or ethambutol. Another $12(26 \%)$ showed favourable responses to drug treatment initially, but eventually relapsed. Four patients had progressive disease while receiving prolonged courses of chemotherapy. Resection was performed in five patients with resultant cure in four. Since the prognosis with drug treatment alone is so unpredictable it is suggested that resection might be part of first line treatment, and that it should usually be performed if patients fail to respond to initial chemotherapy or if they relapse.

Published reports have suggested that pulmonary infection with Mycobacterium xenopi responds to antituberculous chemotherapy and carries a relatively good prognosis for cure..$^{1-3}$ Much of the information on which these views are based, however, has been obtained from small series or case reports on individual patients. Moreover, with the exception of a recent paper by Smith and Citron, ${ }^{4}$ follow up periods have been either short or not

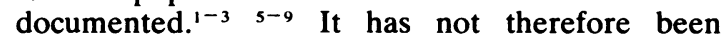
possible to assess accurately the effect of chemotherapy or surgery on the long term prognosis of patients with this condition. We have studied 47 patients with $M$ xenopi infection presenting over a 10 year period in an attempt to assess their response to treatment and prognosis for cure and to identify the forms of treatment most likely to lead to successful outcome.

\section{Methods}

The names of 90 patients whose sputa had yielded

Address for reprint requests: Dr J Banks, Chest Unit, Llandough Hospital, Penarth, S Glam CF6 1XX.

Accepted 4 January 1984 multiple isolates of $M$ xenopi in the years 1970-9 were obtained from the records of the Mycobacterium Reference Unit of the Public Health Laboratory Service in Cardiff, the Public Health Laboratory in Dulwich or the Medical Research Council Unit for Laboratory Studies of Tuberculosis at Hammersmith Hospital, London. Case notes and radiographs were obtained for 61 patients but in the remaining 29 cases the records were either unobtainable or too incomplete for review. From the case notes of the 61 patients reviewed we obtained information about age, sex, pre-existing lung disease, mode of presentation, treatment, and subsequent progress. The chest radiographs were assessed independently by one of the authors, who had no knowledge of the patients' clinical and bacteriological state or treatment.

In vitro sensitivity testing was performed by the "modal" resistance method. ${ }^{10}$ In this method the normal range of tolerance of $M$ tuberculosis to each drug is defined by reference to isolates from new cases of tuberculosis. A fourfold increase above this level is taken as significant resistance. It is convenient to read tests on opportunist mycobacteria in the same way, although a similar correspondence between in vitro sensitivity and therapeutic response 
cannot always be assumed.

Diagnostic criteria are necessary to distinguish those patients with active disease from those in whom $M$ xenopi is a casual contaminant and hence of no clinical significance. To be considered significant multiple isolations of $M$ xenopi were required in the absence of concurrent isolates of other pathogens, including $M$ tuberculosis. Moreover, patients had to have abnormal chest radiographic appearances consistent with mycobacterial disease which could not be attributed to other causes. Fourteen patients failed to fulfill these criteria and were excluded, leaving 47 for review.

The response to treatment was assessed according to the three indices-namely, clinical progress, sputum culture results, and radiographic response. Patients were considered cured if satisfactory clinical progress and radiographic improvement were accompanied by persistently negative sputum cultures. The disease was regarded as stable when the clinical and radiographic pattern remained unchanged irrespective of whether sputum culture results were positive or negative, and progressive if there was clinical and radiographic deterioration with positive sputum cultures.

\section{Results}

Of the 47 patients reveiwed, $39(82 \%)$ were male and the mean age of the group was 61.5 years (range 36-84). Pre-existing lung disease was present in 35 cases $(75 \%)$, of which healed $M$ tuberculosis infection accounted for 14, chronic bronchitis and emphysema 12, and bronchiectasis three; whilst three others had interstitial pulmonary fibrosis (asbestosis, chronic bird fancier's lung, and cryptogenic fibrosing alveolitis). One patient had coalworkers' pneumoconiosis and another intrinsic asthma, and another gave a past history of lobar pneumonia. Two patients were subsequently found to have bronchogenic carcinomas and died while having antituberculous treatment. Lung cancer was diagnosed in a further two patients 10 months and 10 years respectively after completion of their antituberculous chemotherapy. Six patients had previous gastrectomies. In four patients there was no evidence of any pre-existing disorder. Thirty six patients were smokers and in 11 the smoking habits were not documented.

\section{CLINICAL PICTURE}

Forty three patients $(91 \%)$ had respiratory symptoms at the time of diagnosis. In 21 of these the history was characterised by a subacute illness developing during two to four months usually with increasing cough and sputum, weight loss, and malaise. Haemoptysis occurred in eight patients in this group. Another 20 patients gave longer histories of respiratory symptoms, which had often been present for several years, sometimes associated with slowly progressive changes on chest radiographs. These patients were usually referred because of progressive clinical and radiographic deterioration. Haemoptysis was a presenting feature in five of this group. Two patients had an acute illness characterised by repeated haemoptyses and weight loss occurring over a few weeks. Two were symptom free at diagnosis, but were referred after mass miniature radiography. Two patients with interstitial pulmonary fibrosis were found to have new radiographic lesions at follow up but denied new symptoms.

\section{RADIOGRAPHIC ABNORMALITIES}

In 24 patients $(51 \%)$ the intitial chest radiograph showed unilateral disease only. Cavitating disease was present in 45 (96\%).

\section{SENSITIVITY TESTS}

Sensitivity to rifampicin, isoniazid, streptomycin, and $p$-aminosalicylic acid (PAS) was variable and inconsistent (table 1). Furthermore, significant changes in sensitivity to individual drugs-from resistance ratio 8 to fully sensitive and vice versaoccurred in some patients over short periods even when the drug had not been prescribed. This was most noticeable with PAS but also occurred with streptomycin and rifampicin. There were many more cases in which sensitivity patterns changed by a lesser degree during the course of the disease, often from sensitive to borderline resistant (ratio 4) or from ratio 4 to fully resistant (ratio 8 ).

\section{TREATMENT AND RESPONSE}

There had been a variety of treatment regimens and responses to treatment (summarised in tables 2-6). Compliance, so far as it can be judged in a retrospective study, was good.

\section{PATIENTS CURED}

Eleven patients $(23 \%)$ showed satisfactory clinical and bacteriological responses accompanied by radiographic improvement, although only three showed closure of cavities (table 2). The mean follow up period was $\mathbf{3 2}$ months. Six patients received a combination of rifampicin, isoniazid, and ethambutol despite relatively poor in vitro sensitivity results. The addition of second line drugs did not confer any extra benefits.

PATIENTS WHO RELAPSED AFTER AT LEAST NINE MONTHS' CHEMOTHERAPY

Twelve $(26 \%)$ patients (table 3 ) were initially given 
Table 1 Results of in vitro sensitivity tests on initial isolates

\begin{tabular}{|c|c|c|c|c|}
\hline \multirow[t]{2}{*}{ Drug } & \multicolumn{4}{|c|}{ No of patients } \\
\hline & Sensitive & Resistant & Borderline ${ }^{*}$ & Not tested \\
\hline $\begin{array}{l}\text { Streptomycin } \\
\text { Rifampicin } \\
\text { Isoniazid } \\
\text { Ethambutol } \\
\text { Cycloserine } \\
\text { Ethionamide } \\
\text { p-aminosalicylic acid } \\
\text { Capreomycin }\end{array}$ & $\begin{array}{r}33 \\
18 \\
10 \\
1 \\
35 \\
36 \\
4 \\
26\end{array}$ & $\begin{array}{r}7 \\
16 \\
25 \\
38\end{array}$ & $\begin{array}{r}5 \\
13 \\
10 \\
5\end{array}$ & $\begin{array}{r}2 \\
2 \\
3 \\
12 \\
11 \\
7 \\
15\end{array}$ \\
\hline
\end{tabular}

*Ratio 4.

at least nine months' chemotherapy. Most patients had initially received rifampicin and isoniazid combined with either ethambutol or streptomycin despite the in vitro sensitivity patterns, and all improved. All 12 became sputum culture negative but this was accompanied by radiographic improvement in only eight patients. Cavitation persisted in all twelve. Despite these satisfactory initial responses all relapsed at a later date. The results of retreatment were generally poor. Six of the seven patients receiving second courses of chemotherapy developed progressive disease despite treatment with multiple drug regimens chosen according to the in vitro sensitivity. The affected lobes were resected in two of these patients, with resultant cure in each case. Both had unilateral disease radiographically and active disease was present in both resected specimens. The seventh patient who was retreated showed an initial satisfactory response but again relapsed 17 months after the end of treatment. Currently she remains stable while continuing on a third course of treatment, although she has a destroyed, cavitated, fibrosed right upper lobe.

Three of the five patients who were not retreated showed slowly progressive disease, one subsequently dying from his infection. Two others not retreated have remained clinically stable throughout their prolonged follow up, although both have extensively fibrosed and cavitated upper lobes with occasional positive sputum cultures.

Table 2 Treatment regimens given to patients considered cured

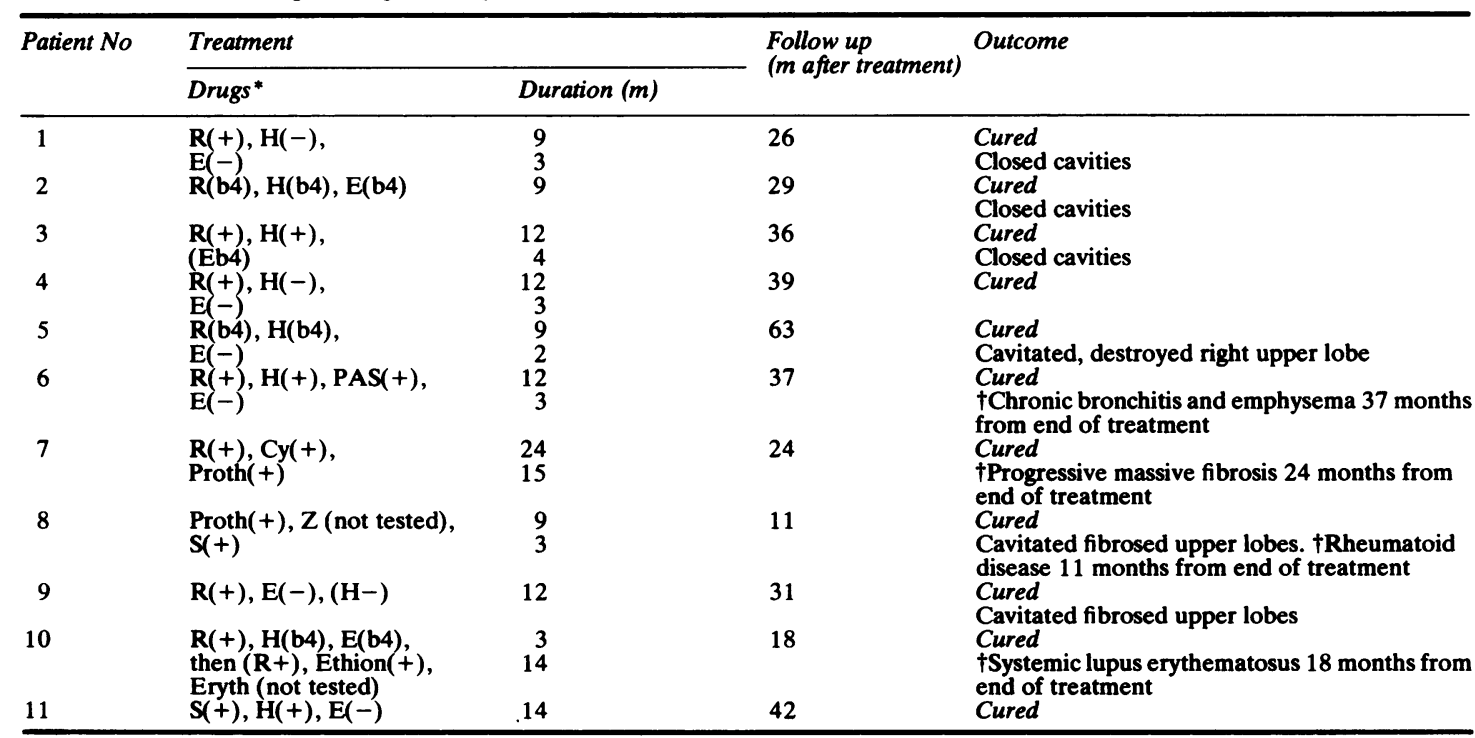

${ }^{*} \mathbf{R}$-rifampicin; H—isoniazid; S—streptomycin; E-ethambutol; PAS—p-aminosalicylic acid; Ethion—ethionamide; Proth— prothionamide; Z-pyrazinamide; eryth-erythromycin; CAP-capreomycin; $\mathrm{Cy}$-cycloserine.,,$+- \mathrm{b} 4$ indicate in vitro sensitive, resistant and borderline resistant. $†$ Died. 
Table 3 Treatment regimens in patients with initial satisfactory responses followed by later relapse

\begin{tabular}{|c|c|c|c|c|c|c|}
\hline \multirow[t]{2}{*}{ Patient No } & \multicolumn{2}{|l|}{ Treatment } & \multirow{2}{*}{$\begin{array}{l}\text { Relapse } \\
\text { (m from end of } \\
\text { initial } \\
\text { treatment) }\end{array}$} & \multicolumn{2}{|l|}{ Retreatment } & \multirow{2}{*}{$\begin{array}{l}\text { Outcome (follow up (m) from end of last } \\
\text { treatment) }\end{array}$} \\
\hline & Drugs* & $\begin{array}{l}\text { Duration } \\
(m)\end{array}$ & & Drugs* & $\begin{array}{l}\text { Duration } \\
(m)\end{array}$ & \\
\hline 12 & $\begin{array}{l}\mathbf{R}(+), \mathbf{H}(-), \mathrm{E}(-) \\
\operatorname{Eryth}(+)\end{array}$ & $\begin{array}{l}18 \\
10\end{array}$ & 4 & $\begin{array}{l}\mathbf{R}(+) \\
\mathbf{H}(-), \mathbf{E}(-) \\
\text { Eryth(+) }\end{array}$ & $\begin{array}{r}15 \\
9 \\
6\end{array}$ & Progressive disease (24) \\
\hline 13 & $\begin{array}{l}\mathbf{R}(-), \mathbf{H}(-) \\
\mathbf{S}(-)\end{array}$ & $\begin{array}{r}12 \\
2\end{array}$ & 8 & $\begin{array}{l}\text { Eryth }(+), C y(+), \\
\text { Ethion(+) }\end{array}$ & 20 & Progressive disease (60) \\
\hline 14 & $\begin{array}{l}\mathbf{R}(+), H(b 4) \\
E(b 4)\end{array}$ & $\begin{array}{r}12 \\
3\end{array}$ & 48 & None & - & Progressive disease (78) \\
\hline 15 & $\begin{array}{l}\mathbf{R}(+), \mathbf{H}(\mathbf{b} 4) \\
\mathrm{E}(-)\end{array}$ & $\begin{array}{r}18 \\
3\end{array}$ & 8 & $\begin{array}{l}\mathbf{S}(+), \mathbf{R}(+), \\
\text { PAS }+(), \\
\text { Ethion(t) }\end{array}$ & 12 & $\begin{array}{l}\text { Progressive disease } \\
\dagger M \text { xenopi (while on treatment) }\end{array}$ \\
\hline 16 & $\mathbf{R}(+), \mathrm{H}(-)$ & 11 & 6 & None & - & Stable disease (culture positive) (66) \\
\hline 17 & $\begin{array}{l}\mathbf{R}(\mathbf{b} 4), \mathrm{E}(-) \\
\mathrm{Cy}(+), \mathrm{H}(\mathrm{b} 4)\end{array}$ & 21 & 6 & None & - & Stable disease (culture positive) (88) \\
\hline 18 & $\begin{array}{l}\mathbf{R}(+), \mathbf{H}(+) \\
\text { PAS }(+)\end{array}$ & $\begin{array}{r}18 \\
1\end{array}$ & 60 & None & - & $\begin{array}{l}\text { Progressive disease } \\
\text { †Myocardial infarction (60) }\end{array}$ \\
\hline 19 & $\begin{array}{l}\mathbf{H}(+), \hat{E}(\mathbf{b} 4) \\
\mathbf{R}(+)\end{array}$ & $\begin{array}{r}12 \\
5\end{array}$ & 56 & $\begin{array}{l}\mathbf{H}(+), \mathbf{R}(+), \\
\mathbf{S}(+), \text { Ethion }(+), \\
\mathrm{E}(\mathrm{b} 4)\end{array}$ & 9 & $\begin{array}{l}\text { Initial improvement. } 2 \text { nd relapse after } 17 \\
\text { months. Retreatment: } \mathbf{R}(+), \mathrm{H}(+), \mathrm{E}(\mathrm{b} 4) \\
\text { Stable disease (culture negative). On } \\
\text { continuing treatment }\end{array}$ \\
\hline 20 & $\mathbf{R}(\mathbf{b} 4), \mathbf{H}(\mathbf{b} 4)$ & $\begin{array}{r}18 \\
6\end{array}$ & 8 & $\begin{array}{l}\mathbf{S}(+), \text { Ethion(+), } \\
\mathbf{H}(-)\end{array}$ & 8 & $\begin{array}{l}\text { Progressive disease-resection } \\
\text { Cure (48) }\end{array}$ \\
\hline 21 & $\mathbf{R}(\mathbf{b} 4), \mathrm{H}(-)$ & 9 & 30 & $\begin{array}{l}\mathrm{Cy}(+), \mathrm{S}(+) \\
\text { Ethion }(+)\end{array}$ & 3 & $\begin{array}{l}\text { Progressive disease-resection } \\
\text { Cure }(42)+\text { Carcinoma oesophagus }\end{array}$ \\
\hline 22 & $\mathbf{R}(\mathbf{b} 4), \mathbf{H}(\mathbf{b} 4)$ & $\begin{array}{r}14 \\
7\end{array}$ & 36 & $\begin{array}{l}\text { R, H, Cy (not } \\
\text { tested) }\end{array}$ & & $\begin{array}{l}\text { Progressive disease } \\
+M \text { xenopi }(60)\end{array}$ \\
\hline 23 & $\begin{array}{l}\text { H(t), PAS(+), } \\
\text { S(+), } \\
\text { Cy(t), Ethion }(+), \\
\text { R(+), } \\
\text { CAP(+) }\end{array}$ & $\begin{array}{r}2 \\
30 \\
3\end{array}$ & 10 & None & - & $\begin{array}{l}\text { Progressive disease } \\
\text { †M xenopi (36) }\end{array}$ \\
\hline
\end{tabular}

*Abbreviations and symbols as in table 2.

†Died.

Table 4 Responses in patients who received less than nine months' treatment

\begin{tabular}{|c|c|c|c|}
\hline \multirow[t]{2}{*}{ Patient No } & \multicolumn{2}{|l|}{ Treatment } & \multirow[t]{2}{*}{ Outcome (follow up $(m)$ ) } \\
\hline & Drugs* & Duration (m) & \\
\hline 24 & $\begin{array}{l}\mathbf{R}(+), \mathbf{H}(\mathbf{b} 4) \\
\text { Ethion }(+)\end{array}$ & $\begin{array}{l}6 \\
2\end{array}$ & $\begin{array}{l}\text { Initial improvement. Relapsed after } 3 \text { months; not } \\
\text { retreated. Progressive disease. †Myocardial } \\
\text { infarction (9) }\end{array}$ \\
\hline 25 & $\begin{array}{l}\mathbf{R}(+), \mathbf{H}(\mathbf{b} 4) \\
\mathrm{E}(-)\end{array}$ & $\begin{array}{l}6 \\
3\end{array}$ & Stable disease (36). Culture positive \\
\hline 26 & $\begin{array}{l}\mathbf{R}(+), C y(+), \text { Proth(+) } \\
\mathbf{S}(+)\end{array}$ & 2 weeks & $\begin{array}{l}\text { Initial improvement. Relapsed after } 11 \text { months; } \\
\text { retreated with } \mathrm{R}(+), \mathrm{Cy}(+) \text {, Prothion }(+), \mathrm{CAP}(+) \\
\text { for } 3 \text { months, but progressive disease on } \\
\text { treatment. } \\
+M \text { xenopi confirmed at necropsy and lung } \\
\text { cancer (10) }\end{array}$ \\
\hline $\begin{array}{l}27 \\
28\end{array}$ & $\begin{array}{l}\mathbf{R}(-), \mathbf{E}(-), \mathbf{H}(-) \\
\mathbf{R}(-), \mathbf{H}(-), \mathbf{E}(-)\end{array}$ & $\begin{array}{l}2 \\
8\end{array}$ & $\begin{array}{l}\text { Progressive disease (48) } \\
\text { Clinical and radiological response on treatment } \\
\dagger \text { Peptic ulcer ( } 8 \text { months on treatment) }\end{array}$ \\
\hline 29 & $\begin{array}{l}\mathbf{R}(-), \text { PAS(-), E(b4) } \\
\mathbf{S}(+)\end{array}$ & $\begin{array}{l}2 \\
1\end{array}$ & $\begin{array}{l}\text { Progressive disease } \\
\dagger M \text { xenopi (24) }\end{array}$ \\
\hline $\begin{array}{l}30 \\
31\end{array}$ & $\begin{array}{l}\mathbf{H}(+), \mathbf{S}(+), \text { Ethion }(+), \text { CAP/E (not tested) } \\
\text { E(-), PAS (not tested) } \\
\mathbf{R}(+)\end{array}$ & $\begin{array}{l}5 \\
2 \\
3 \text { weeks }\end{array}$ & $\begin{array}{l}\text { †Lung cancer ( } 5 \text { months on treatment) } \\
\text { Progressive disease } \\
\text { †M xenopi ( } 24)\end{array}$ \\
\hline 32 & $\mathbf{R}(+), \mathbf{H}(+), \mathbf{S}(+), \mathbf{Z}$ (not tested) & $<2$ & $\begin{array}{l}\text { Progressive disease } \\
\dagger M \text { xenopi and lung cancer, on antituberculous } \\
\text { treatment }\end{array}$ \\
\hline 33 & $\mathbf{R}(\mathbf{b} 4), \mathrm{H}(\mathrm{b} 4)$, Prothion(+), $\mathrm{Cy}(+)$ & 2 & $\begin{array}{l}\text { Progressive disease } \\
+M \text { xenopi }(2)\end{array}$ \\
\hline 34 & $\mathbf{R}(+), \mathbf{H}(+), \mathrm{E}(-)$ & 1 & †Femoral embolus and amputation (on treatment) \\
\hline
\end{tabular}

*Abbreviations and symbols as in table 2.

†Died. 
Table 5 Responses in patients receiving initially less than nine months' chemotherapy followed later by more prolonged treatment

\begin{tabular}{|c|c|c|c|c|c|c|}
\hline \multirow[t]{2}{*}{ Patient No } & \multicolumn{2}{|l|}{ Initial treatment } & \multirow[t]{2}{*}{ Outcome } & \multicolumn{2}{|l|}{ Retreatment } & \multirow[t]{2}{*}{ Outcome (follow up (m)) } \\
\hline & Drugs* & $\begin{array}{l}\text { Duration } \\
\text { (m) }\end{array}$ & & Drugs* & $\begin{array}{l}\text { Duration } \\
\text { (m) }\end{array}$ & \\
\hline 35 & $\begin{array}{l}\mathbf{R}(+), \mathbf{H}(+) \\
\mathbf{S}(+), \\
\text { Ethion(+) }\end{array}$ & $\begin{array}{l}5 \\
11 / 2 \\
2^{1 / 2}\end{array}$ & $\begin{array}{l}\text { Progressive disease over } \\
11 \text { years }\end{array}$ & $\begin{array}{l}\mathbf{R}(+), \mathbf{H}(-) \\
\mathbf{E}(-)\end{array}$ & $\begin{array}{l}9 \\
3\end{array}$ & Cured (12) \\
\hline 36 & $\begin{array}{l}\mathbf{R}(+), \mathrm{E}(-), \\
\mathbf{H}(\text { not tested) }\end{array}$ & 3 & \multirow{3}{*}{$\begin{array}{l}\text { Progressive disease over } \\
10 \text { months } \\
\text { Progressive disease over } \\
12 \text { months } \\
\text { Relapsed } 2 \text { years later }\end{array}$} & $\mathbf{R}(+)$, Eryth(+) & 18 & Cured (40) \\
\hline 37 & $\mathbf{R}(\mathbf{b} 4), \mathbf{H}(\mathbf{b} 4)$ & 2 & & \multirow{2}{*}{$\begin{array}{l}\mathbf{R}(\mathbf{b} 4), \mathbf{H}(\mathbf{b} 4) \\
\mathbf{S}(+) \\
\mathbf{R}(+), \mathbf{H}(-) \\
\text { Prothion(+), Z (not tested) }\end{array}$} & \multirow{2}{*}{$\begin{array}{l}9 \\
2 \text { weeks } \\
12 \\
6\end{array}$} & Cured (42) \\
\hline 38 & $\begin{array}{l}\mathbf{R}(+), \mathbf{H}(-), \\
\mathbf{E}(-)\end{array}$ & 6 & & & & $\begin{array}{l}\text { Cured but } 2 \text { nd relapse } 8 \\
\text { months later. 3rd treatment: } \\
\mathbf{R}(+), \text { Proth }(+), \text { Cy }(+) \\
9 \text { months. Stable disease: } \\
\text { (sputum positive) }\end{array}$ \\
\hline
\end{tabular}

*Abbreviations and symbols as in table 2.

PATIENTS RECEIVING LESS THAN NINE MONTHS' CHEMOTHERAPY

Eleven patients (table 4) received less than nine months' chemotherapy. The eventual outcome was unfavourable in all cases. Five showed progressive disease, four eventually dying with active infection, although one was found to also have a bronchogenic carcinoma at necropsy. Three others died from unrelated causes while receiving treatment. Of the remaining three, two showed initial improvement while receiving treatment for six months and four months respectively but relapsed with progressive disease within months of stopping chemotherapy. The remaining patient has stable disease three years after treatment.

A further four patients initially received less than nine months' treatment, but were later treated for more prolonged periods, with variable responses (table 5). One patient had progressive disease during the 11 years following the initial treatment and more recently has received a second course of treatment with rifampicin, isoniazid, and ethambutol with resultant cure, although follow up has been for only one year.

\section{PATIENTS NOT RESPONDING TO INITIAL CHEMOTHERAPY}

Five other patients failed to respond to initial chemotherapy (table 6). Four had progressive disease while receiving prolonged courses of treatment with multiple appropriate drug regimens derived from results of in vitro sensitivity tests. One patient had early resection followed by nine months' chemotherapy with resultant cure, and has remained well for the seven years since operation. A second patient was eventually cured after resection but the other two died from their disease after the end of chemotherapy. The fifth patient is considered cured but has been having treatment for five years so far.

PATIENTS WHO DID NOT HAVE CHEMOTHERAPY Four patients (not tabulated) received no

Table 6 Outcome in patients who deteriorated while receiving chemotherapy

\begin{tabular}{|c|c|c|c|}
\hline \multirow[t]{2}{*}{ Patient No } & \multicolumn{2}{|l|}{ Treatment } & \multirow[t]{2}{*}{ Outcome } \\
\hline & Drugs* & Duration (m) & \\
\hline 39 & $\mathbf{H}(-), \mathbf{S}(+)$, Ethion(+) & $\begin{array}{r}8 \\
17 \\
24\end{array}$ & $\begin{array}{l}\text { Poor response-cycloserine added. Cured while continuing treatment } \\
\text { for } 60 \text { months }\end{array}$ \\
\hline 40 & $\mathbf{R}(+)$, Ethion $(+), \mathrm{H}(+)$ & 14 & $\begin{array}{l}\text { Progressive disease on treatment. } \dagger M \text { xenopi } 14 \text { months from end of } \\
\text { treatment }\end{array}$ \\
\hline 41 & $\mathrm{Cy}(+)$, Ethion $(+), \mathrm{H}(-)$ & 1 & $\begin{array}{l}\text { Progressive disease. Resected after } 1 \text { month's chemotherapy } \\
\text { Chemotherapy continued for } 9 \text { months after resection. Cure-follow } \\
\text { up } 84 \text { months }\end{array}$ \\
\hline 42 & $\begin{array}{l}\mathbf{R}(+), \mathbf{H}(-) \\
\text { S(+), Ethion(+), } \\
\text { Eryth(+) }\end{array}$ & $\begin{array}{r}11 \\
13 \\
9\end{array}$ & $\begin{array}{l}\text { Progressive disease. Resection after } 13 \text { months' chemotherapy } \\
\text { Cure-follow up } 45 \text { months }\end{array}$ \\
\hline 43 & $S(+), R(+), H(b 4), E(b 4)$ & 4 & Progressive disease. $† M$ xenopi several months from end of treatment \\
\hline
\end{tabular}

*Abbreviations and symbols as in table 2 .

+Died. 
chemotherapy and the disease progressed in all. One patient deteriorated over a period of three years and was then submitted to lobectomy. She was known to suffer from chronic bronchitis. She developed postoperative bronchopneumonia and died two weeks later. A second patient is alive but has deteriorated with progressive disease during four years and a third patient deteriorated for 10 years, eventually dying from lung cancer. The fourth patient had progressive disease for 12 months and died from a cerebrovascular accident.

\section{Discussion}

Some reports have suggested that infection with $M$ xenopi is relatively easy to treat and carries a good prognosis for cure because the organism is usually sensitive in vitro to several antituberculous drugs. ${ }^{1-3}$ Treatment with rifampicin, isoniazid, and streptomycin or rifampicin and isoniazid with a suitable second line drug has been advocated. ${ }^{2}$ "In contrast to these reports, we found that in vitro susceptibility patterns to rifampicin, isoniazid, and streptomycin varied and were inconsistent, although the organisms were sensitive to cycloserine and ethionamide whenever tested (table 1). The changes in susceptibility to certain drugs (which could occur spontaneously even though the drug was not being used in treatment) may have arisen as a result of reinfection or could possibly reflect the limitation of in vitro laboratory testing with this organism. Others have reported changes in drug sensitivity which have occurred during the course of the disease. ${ }^{12}$ Moreover, several patients in our series showed poor correlation between in vitro sensitivity results and clinical response. It is therefore questionable whether treatment should be tailored according to in vitro sensitivity results.

We found that the response to treatment, particularly in the long term, was completely unpredictable. Some patients had no response to initial treatment, deteriorating while receiving chemotherapy. Most patients, however, felt better, became sputum culture negative, and showed radiographic improvement (although cavities were rarely closed completely) while on treatment. But such favourable results in the short term, as reported by others, ${ }^{23589}$ can be misleading since there is a tendency for the disease to relapse, sometimes slowly and insidiously over several years. This occurred in $26 \%$ of patients who received treatment, relapse occurring up to five years after cessation of treatment. Possibly some patients shown in table 2 as cured may relapse at a future date and those patients regarded as having stable disease may in fact have slowly progressive disease. Response to retreatment was generally poor. These points emphasise the need for careful and prolonged follow up in those patients considered cured.

Some patients, however, do appear to be cured with chemotherapy. In a recent review by Smith and Citron, eight of 11 patients given chemotherapy were considered cured after follow up period of at least three years. ${ }^{4}$ Seven of these initially had cavities, which closed in five cases after chemotherapy (unpublished observations). Two patients in their series, however, had no response despite receiving two or more drugs to which the organism showed in vitro sensitivity; and a third patient relapsed one year after the end of treatment. Costrini et al, reviewing a series of 19 patients, report a variable response to antituberculous therapy. ${ }^{13}$ Most patients in their series received isoniazid and ethambutol and they concluded that treatment with standard antituberculous drugs was generally successful. Three patients, however, showed progressive disease radiologically while receiving teatment and a fourth improved radiologically but later relapsed. Five other patients failed to show any radiological improvement. The bacteriological responses in these individual patients are not described but three of 15 patients remained culture positive after treatment.

In our series $23 \%$ of patients have not so far relapsed and fulfil our criteria for cure. Most of these patients had been treated with rifampicin and isoniazid combined with either streptomycin or ethambutol. There were no features which allowed separation of the patients who were to do well from those who had an unfavourable outcome; in particular, neither the mode of onset nor the presence or absence of pre-existing disease were useful as predictors of response. Possibly there was some impairment of immune response in those patients who had unfavourable responses to treatment, although we are not aware of any experimental evidence on this point. Impaired immunological responses may also be important in the pathogenesis of invasive disease as well as in the persistence of simple colonisation with the organism.

We were unable to determine an adequate period of treatment, but it was apparent that those patients who received chemotherapy for less than nine months did not do well. Furthermore, a failure to treat was always followed by progressive disease, which in one patient continued for 10 years.

Resection was performed because of failure to respond to chemotherapy or because of subsequent relapse in four patients, with resultant cure in all, the follow up ranging from three and a quarter to seven years. The fifth patient undergoing surgery died two weeks later from postoperative pneumonia. She had 
deteriorated with untreated progressive disease during the three years preceding surgery and suffered from chronic bronchitis. Since the prognosis with drug treatment alone is so unpredictable in this condition there would seem to be a good argument for resection in some patients, particularly those who fail to respond initially to chemotherapy or who relapse. Earlier intervention while the disease remains limited is likely to carry a better chance of success. Even so, coexistent pulmonary disease or other conditions may often preclude operative management in these patients.

\section{References}

${ }^{1}$ Koizumi JH, Sommers HM. Mycobacterium xenopi and pulmonary disease. Am J Clin Pathol 1980;73:82630.

${ }^{2}$ Boguerts Y, Elinck W, Van Renterghem D, Pauwels R, Van Der Straeten M. Pulmonary disease due to Mycobacterium xenopi. Report of two cases. Eur J Respir Dis 1982;63:298-304.

${ }^{3}$ Tellis CJ, Beechler CR, Chashi DK, Fuller SA. Pulmonary disease caused by Mycobacterium xenopi. Two case reports. Am Rev Respir Dis 1977;116:779-83.

${ }^{4}$ Smith MJ, Citron KM. Clinical review of pulmonary disease caused by Mycobacterium xenopi. Thorax $1983 ; 38: 373-7$.
${ }^{5}$ Richter PE, Tomasovic AA, Paxon TG. Pulmonary disease related to Mycobacterium xenopi. Med J Aust 1969;i: 1246-7.

' Engbaek HC, Jergmann B, Bases I, Will DW. M xenopi. A bacteriological study of $M$ xenopi including case reports of Danish patients. Acta Pathol Microbiol Scand 1967;69:576-94.

${ }^{7}$ Thibier R, Vivien JN, Lepeuple A. Sept cas de pleuropneumopathie a "Mycobacterium xenopi." Rev Tuberc Pneumol 1970;34:623-5.

${ }^{8}$ Elston HR, Duffy JP. Mycobacterium xenopi and mycobacteriosis. A clinical and bacteriological report. Am Rev Respir Dis 1973;108:944-9.

${ }^{9}$ Fields BT Jr, Bishop MC, Brosbe EA, Bates JH. Pulmonary disease caused by Mycobacterium xenopi and Histoplasma capsulatum. A case report. Am Rev Respir Dis 1969:99:590-4.

${ }^{10}$ Marks J. A system for the examination of tubercle bacilli and other mycobacteria. Tubercle 1976;57:207-25.

$"$ Wolinsky E. Non-tuberculous mycobacteria and associated diseases. Am Rev Respir Dis 1979;119:107-59.

12 McDonald PJ, Tomasovic AA, Evans C. Mycobacterium xenopi pulmonary infection in man. Med $J$ Aust 1972;i:873.

${ }^{13}$ Costrini AM, Mahler DA, Gross WM, Hawkins JE, Yesner R, D'Esopa ND. Clinical and roentgenographic features of nosocomial pulmonary disease due to Mycobacterium xenopi. Am Rev Respir Dis 1981;123: 104-9. 